\title{
Strategic Analysis for the Installation of Field Hospitals for COVID-19 Control: An Approach Based on P-Median Model
}

\author{
Leandro de Mattos Bento Soares ${ }^{a}$, Miguel Ângelo Lellis Moreira ${ }^{\mathrm{a}, \mathrm{b}, 1}$, \\ Marcio Pereira Basilio ${ }^{c}$, Carlos Francisco Simões Gomes ${ }^{b}$, \\ Marcos dos Santos ${ }^{\mathrm{a}, \mathrm{d}}$, and Igor Pinheiro de Araújo Costa ${ }^{\mathrm{a}, \mathrm{b}}$ \\ ${ }^{a}$ Naval Systems Analysis Center, Rio de Janeiro, RJ 20091-000, Brazil \\ ${ }^{b}$ Fluminense Federal University, Niterói, RJ 24210-240, Brazil \\ ${ }^{c}$ Military Police of the State of Rio de Janeiro, Rio de Janeiro, 20031-040, Brazil \\ ${ }^{d}$ Military Institute of Engineering, Urca, RJ 22290-270, Brazil
}

\begin{abstract}
Concerning the expansion of the coronavirus in the world, the search for the development of solutions related to the control and mitigation of the pandemic situation became constant. The paper addresses an analysis of localities for the installation of field hospitals, highly requested as a point of treatment for COVID-19. In this scenario, a framework based on the P-median approach and mathematical programming is proposed, enabling an optimization model as an analysis format for the problematic situation. To support the implementation of the model, a computational tool for data processing was developed, integrating an optimization model to the geographical evaluation, exploring in the analysis numerical and graphical resources. As a validation of the study, a case study in the city of Rio de Janeiro - Brazil is presented, analyzing 162 neighborhoods and determining seven favorable localities for the installation of field hospitals.
\end{abstract}

Keywords. P-median, Mathematical Programming, Computational Tool.

\section{Introduction}

The COVID-19 global pandemic was easily spread by the proximity of people, especially in large agglomerations, a common characteristic of big cities and metropolitan regions [1]. Since its dissemination, the coronavirus has highlighted a series of weaknesses in the health systems around the world, highlighting the problems in accessing the essential resources needed to face this pandemic, such as the lack of respirators and protective equipment [2].

Regarding the expansion of the disease, facilitated by its transmission through contact and movement of people, it was necessary to adopt isolation measures that help in the control and mitigation of virus transmission [3]. In this context, the search for alternative solutions motivated the development of studies in the most diverse areas of science, in search of providing knowledge that would make prevention, prediction, and consequently the minimization and control of the COVID-19 pandemic [4].

${ }^{1}$ Corresponding Author, Miguel Ângelo Lellis Moreira, Production Department, Fluminense Federal University, Niterói, RJ 24210-240, Rio de Janeiro, Brazil; E-mail: miguellellis@id.uff.br. 
In this scenario, in many cities, there was a need to create field hospitals based on temporary facilities to carry out diagnostic tests, hospitalization, and initiation of early treatments for the population, searching to mitigate the problems related to the given pandemic moment [5]. Based on this fact, was made the following question: How to define a favorable location for the installation of field hospitals, minimizing circulation of the population, and providing access to medical care?

Concerning the questioning, the Operational Research (OR) provides, through its approaches and methodologies, the analysis of complex problems with the technical and scientific basis, providing the structuring, understanding, and, in specific cases, the viability of an optimal or favorable solution for a given problem [6].

The problem of optimal allocation of resources is one of the main areas of study present in OR, thus mathematical programming models, aimed at optimizing resources, minimizing or maximizing, make it possible to adapt to the problem in question [7]. In this context, the given models can work as an aid in the clarification of favorable points for the installation of campaign hospitals based on a set of restrictions present in the context and location under analysis.

Regarding the scenario presented, the study addresses the evaluation of localities in the city of Rio de Janeiro - Brazil for the installation of field hospitals as an aid in the control of the COVID-19 pandemic. For the analysis, an optimization model based on P-median modeling is provided, enabling the identification of favorable localities to install the field hospitals. In search to support the implementation, a computational framework based on R software [8] was developed, making possible the implementation of the optimal algorithm, by the moment that dealing with the analysis of big data sets, computational tools make possible not only to explore numerical resources but also graphical resources in favor of a more interactive analysis with the decision-maker [9].

The paper is structured into four sections. After the introduction, section 2 presents the developed framework, exploring the mathematical modeling and computational resources. Section 3 presents the case study, detailing the problematic situation and data set and exploring the steps of the numerical procedure and the results analysis. Section 4 concludes the study, exposing the gains of the approaching and proposal for future works.

\section{Framework Model Analysis}

As discussed in the introduction, the framework presents the integration between mathematical programming, data processing, and graphical analysis. The computational tool was developed in $\mathrm{R}$ language [8], which stands out among technologies based on mathematical and statistical applications, enabling to perform numerical and graphical analysis through your packages and tools [10].

The proposed approach allows for flexibility regarding the restrictions inputted in the mathematical programming model. However, it is worth mentioning that, in general, it uses modeling to solve the P-median problem. It is noteworthy that the framework, is restricted to the geographical dataset, that is, the input data consists of latitudinal and longitudinal points.

The script provides a simple and intuitive interaction, firstly, the structuring and conversion of the dataset based on latitudinal and longitudinal data is performed. Then, the mathematical modeling of the problem to implement the optimization model is realized. In the end, the results through numerical tables and geographical graphics are exposed. Figure 1 presents the flowchart concerning the framework procedure. 


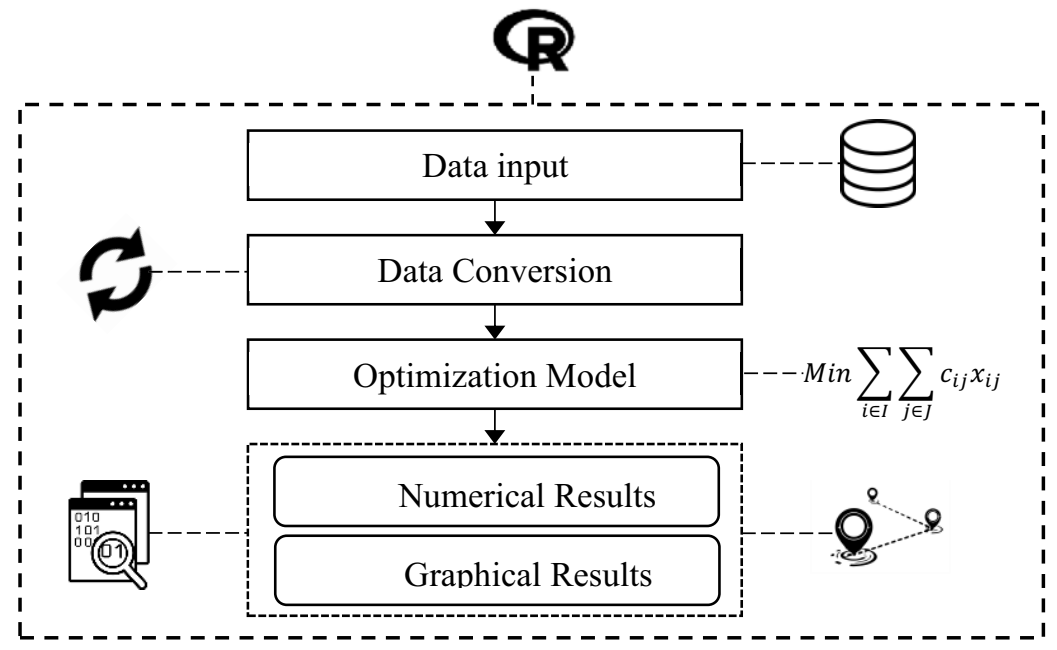

Figure 1. Framework optimal model

\subsection{Data Processing}

The given step consists of the conversion of the dataset to numerical rating scores to set up the optimization model. Thus, initially, a dataset (.csv) based on the information presented in table 1 is imported, exposing all possible installation points, describing their location and geographic coordinates. Considering that the framework is related to the analysis of the COVID-19 pandemic situation, the number of infected cases of each region works as a weighting factor for the problem.

Table 1. Data set input description

\begin{tabular}{ccccc}
\hline Facilities & Localities & Latitude & Longitude & Covid Cases \\
\hline Field Hospital & Place $_{1}$ & Lat $_{1}$ & Long $_{1}$ & $n c_{1}$ \\
Field Hospital $_{2}$ & Place $_{2}$ & Lat $_{2}$ & Long $_{2}$ & $n c_{2}$ \\
Field Hospital $_{n}$ & Place $_{n}$ & Lat $_{n}$ & Long $_{n}$ & $n c_{n}$ \\
\hline
\end{tabular}

Localization problems can arise in two different scopes: the plane and the network, considering the necessity to express the distance between a pair of points. The network handles all possible paths between each pair of points, which may eventually be affected by topographical elements, barriers, and oriented paths. On the other hand, the plane uses the concept of Euclidean distances, which defines the shortest distance between two points based on a straight line connecting them [11].

Thus, as presented in [12], to define the Euclidean distance of $(A, B)$, given by the points $A=\left(x_{a}, y_{\mathrm{a}}\right)$ and $B=\left(x_{b}, y_{b}\right)$, considering that the segment formed by points $A$ and $B$ is not parallel to any of the axes, it is used a point $C=\left(x_{b}, y_{a}\right)$, and points $A, B$ and $C$ become vertices of a right triangle in $C$, applying the Pythagorean Theorem (1).

$$
d_{a b}(A, B)=\sqrt{\left(x_{b}-x_{a}\right)^{2}+\left(y_{b}-y_{a}\right)^{2}}
$$

Based in the given problematic, the equation (1) is operated by latitudinal and longitudinal points, as presented in figure 2 . The number of covid cases weights the function, generating a rating scores $\left(c_{a b}\right)(2)$ for each variable of optimization problem. 


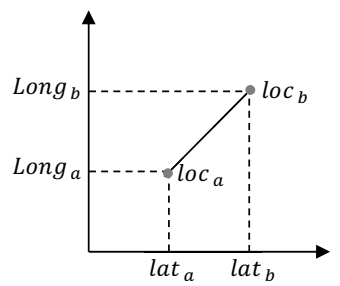

Figure 2. Distance between two localities

$$
c_{a b}=d_{a b}(A, B) n c_{b}
$$

Obtained the rating scores $\left(c_{a b}\right)$ between the pairs of locations, a matrix of variables (3) is generated, exposing the respective costs among all locations of the problem.

$$
\left(\begin{array}{ccccc}
c_{11} & c_{12} & c_{13} & \cdots & c_{1 n} \\
c_{21} & c_{22} & c_{23} & \cdots & c_{2 n} \\
c_{31} & c_{32} & c_{33} & \cdots & c_{3 n} \\
\vdots & \vdots & \vdots & \ddots & \vdots \\
c_{n 1} & c_{n 2} & c_{n 3} & \cdots & c_{n n}
\end{array}\right)
$$

\subsection{Optimization Model}

Based on the P-median model [13], the modeling consists of a mathematical model of integer programming, in a binary format, searching for determining optimal points within a set of constraints $[14,15]$, as exposed by system (4).

$$
\operatorname{Min} \sum_{i \in I} \sum_{j \in J} c_{i j} x_{i j}
$$

s.t.

$$
\begin{array}{ll}
\sum_{i \in I} x_{i j}=1 & \forall_{j} \in J \\
x_{i j} \leq y_{i}, \quad \forall_{i} \in I, \quad \forall_{j} \in J \\
\sum_{i \in I} y_{i}=P \\
\quad x \in B^{|I||J|}, \quad y \in B^{|I|}
\end{array}
$$

In the given system, the objective function minimizes the total rating scores of the evaluation, indicating the minimum possible agglomeration of the population to access a field hospital. The restrictions certify that each location will be served by only one location, and can serves itself. The variable $y_{i}$ presents the number of points available for service, limited to $P$, which indicates the maximum possible installations.

Concerning the computational tool, the Lpsolve package [16] was implemented, making possible to perform optimal algorithms based on mathematical programming.

\subsection{Model of Result Analysis}

In search to provide a robustness analysis, integrated with numerical resources, it is enabled on the framework a geographical analysis of problematic. Through the implementation of Leaflet package [17], it is possible to plot the points in evaluation in a real map, built by geographic data.

After provide the optimal results, the favorable points for facilities are exposed in the map, highlighting the facility centers and their respective locations for access, providing an interactive graphical analysis of results. 


\section{Case Study}

Using the city of Rio de Janeiro as a region of analysis for the given problem a set of neighborhoods that provide the installation of a limited number of campaign hospitals in the fight against COVID-19 is considered. All data presented in the study were provided through data repositories of the Brazilian Ministry of Health, and are available for public access (https://covid.saude.gov.br/).

In the evaluation, 162 locations were considered, where their respective geographic coordinates using the Google Earth Pro software were obtained. For study purposes, the data regarding the numbers of infected in each region in a constant format is performed. Table 2 presents partially the set of inputs.

Table 2. Partial set of input

\begin{tabular}{|c|c|c|c|c|}
\hline Facilities & Localities & Latitude & Longitude & Covid Cases \\
\hline Field Hospital ${ }_{1}$ & Abolição & -22.888002 & -43.299279 & 289 \\
\hline Field Hospital ${ }_{2}$ & Acari & -22.825531 & -43.344460 & 177 \\
\hline 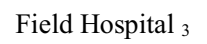 & Água Santa & -22.905568 & -43.308167 & 180 \\
\hline$\vdots$ & $\vdots$ & $\vdots$ & $\vdots$ & $\vdots$ \\
\hline Field Hospital 162 & Vista Alegre & $-22,8303$ & $-43,3167$ & 365 \\
\hline
\end{tabular}

Based on the set of inputs, the algorithm developed (Figure 1) establishes the variable costs, representing the weighted value of Euclidean distances by the number of infected cases per region, as presented in section 2.1. Table 3 presents the partial matrix of costs generated for each pair of locations.

Table 3. Partial set of costs between each pair of localities

\begin{tabular}{rccccccc}
\hline \multicolumn{1}{c}{ Localities } & Abolição & Acari & Água Santa & Anchieta & \multicolumn{2}{c}{$\ldots$} & Vista Alegre \\
\hline Abolição & 0 & 13.6 & 3.50 & 102.7 & $\ldots$ & 5.60 \\
Acari & 22.3 & 0 & 15.8 & 45.60 & $\ldots$ & 6.70 \\
Água Santa & 5.70 & 15.6 & 0 & 106.4 & $\ldots$ & 6.30 \\
Anchieta & 34.2 & 9.30 & 22.1 & 0 & $\ldots$ & 8.80 \\
$\vdots$ & $\vdots$ & $\vdots$ & $\vdots$ & $\vdots$ & $\ddots$ & $\vdots$ \\
Vista Alegre & 40.4 & 29.7 & 28.1 & 41.3 & $\ldots$ & 0 \\
\hline
\end{tabular}

In search to determine the optimal locations for the installation of field hospitals, the optimization model was applied. Through the framework, 26406 variables $\left(x_{i j}\right)$ corresponding to 162 localities $\left(y_{i}\right)$ were generated. In the evaluated scenario, a maximum of seven field hospitals $(P=7)$ were considered.

After the processing of the optimal model, the seven favorable localities to the installation of field hospitals at Rio de Janeiro were defined. Table 4 exposes the optimal localities, along with the number and percentage of access localities.

Table 4. Partial set of input

\begin{tabular}{lccc}
\hline \multicolumn{1}{c}{ Facilities } & Localities & locations served $\left(\mathrm{n}^{\circ}\right)$ & locations served $(\%)$ \\
\hline Field Hospital $_{7}$ & Anil & 18 & $11.1 \%$ \\
Field Hospital $_{39}$ & Cosme Velho & 26 & $16 \%$ \\
Field Hospital $_{68}$ & Inhoaiba & 13 & $8 \%$ \\
Field Hospital $_{72}$ & Jacaré & 33 & $20.37 \%$ \\
Field Hospital $_{77}$ & Jardim Carioca & 15 & $9.26 \%$ \\
Field Hospital $_{93}$ & Marechal Hermes & 31 & $19.13 \%$ \\
Field Hospital $_{155}$ & Vila da Penha & 26 & $16 \%$ \\
\hline
\end{tabular}


For obtained results, it was possible to generate the graphical analysis by geographical map. As presented in figure 3, it was defined the seven optimal locations, each one represented for one color. The center point of each colored circle represents the optimal locality, and the colored points marked on the map represent the other localities of the evaluated set, that should access the optimal location for their respective color.

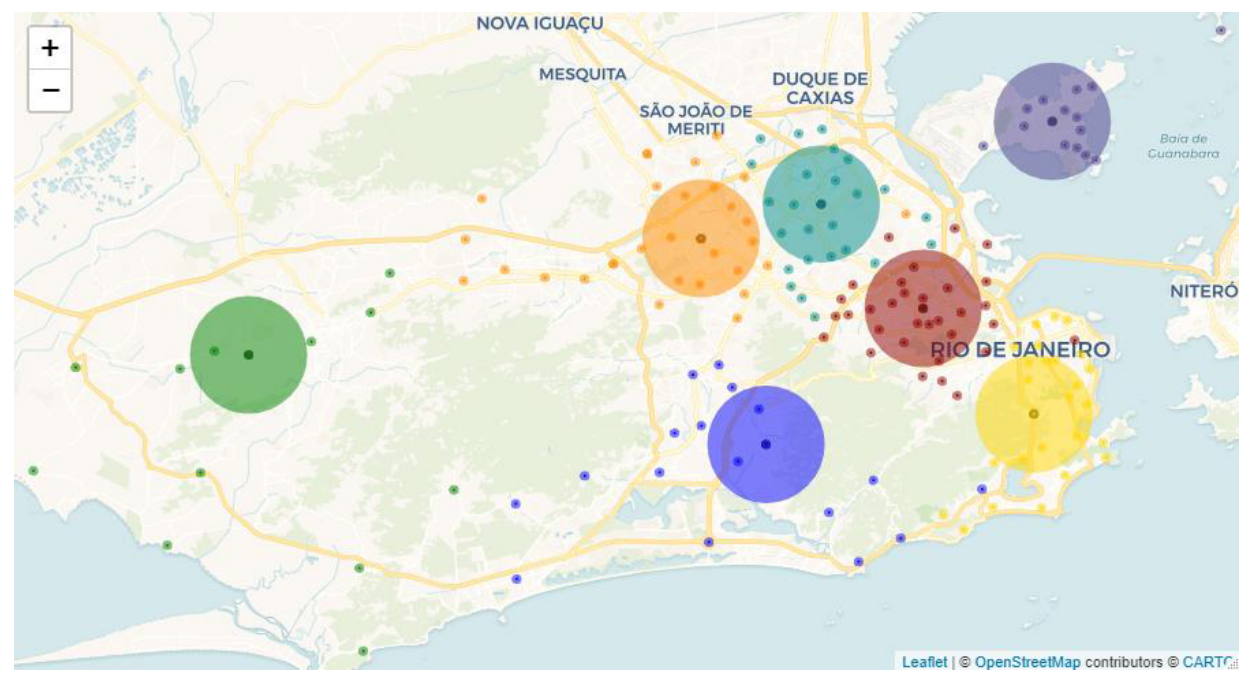

Figure 3. Graphical results of the optimal localities

Considering the rating scores to be minimized $\left(c_{a b}\right)$, representing a relative agglomeration of people to access field hospitals, in the evaluation a total value of 1663.1 was obtained. In addition to the analysis, three other analyses, now considering a maximum $(P)$ of 10, 15, and 20 field hospitals, resulting in rating scores of 1307, 923.5, and 719.7 , respectively, were carried out. In this scenario, is understood the decreasing of rating scores by the increase of installation of field hospitals.

With the given analysis, it is observed that in addition to making it possible to determine optimal locations, it is also possible to clarify a favorable number of field hospitals, searching to find a balance point for a given total number of hospitals.

Based on the realized analysis, it is necessary to emphasize that the given model, in addition to providing a numerical, geographical, and graphical analysis based on the P-median problem, can be adapted to different case studies and data sets, with the need to obtain the coordinates geographic locations of a given problem.

\section{Conclusion}

The study approaches the analysis of localities for the installation of field hospitals in the fight against COVID-19, using the P-median modeling. In a case study, the city of Rio de Janeiro was used as a basis for evaluation, identifying the possible neighborhoods to be used in the construction of facilities concerning a pandemic situation.

For a given analysis, the creation of rating scores variables indicating a relative agglomeration of the population to access a given medical care point was used. It is noteworthy that the given model brings an approximate analysis, with updates in future studies, however, it can be of great value for decision-making scenarios. 
A computational tool to support the evaluation process was developed, enabling the conversion of geographical data to build an optimization model based on mathematical programming and the P-median model. Based on the results generated by the optimization model, numerical and graphical resources of result analysis were explored.

In this context, it concludes that the given study is favorable to the analysis of localities for facilities, and the computational framework can be adapted to different types of analyzes and problems. For future works, we search to integrate other constraining factors into the optimization model, making it possible to consider different service capabilities, and other resource limitations providing a closer approximation of the model to reality.

\section{References}

[1] Alsaeedy AAR, Chong EKP. Detecting Regions At Risk for Spreading COVID-19 Using Existing Cellular Wireless Network Functionalities. IEEE Open Journal of Engineering in Medicine and Biology 2020;1:187-9. https://doi.org/10.1109/ojemb.2020.3002447.

[2] de Sutter A, Llor C, Maier M, Mallen C, Tatsioni A, van Weert H, et al. Family medicine in times of 'COVID-19': A generalists' voice. European Journal of General Practice 2020;26:58-60. https://doi.org/10.1080/13814788.2020.1757312.

[3] Costa IP de A, Sanseverino AM, Barcelos MR dos S, Belderrain MCN, Gomes CFS, Santos M dos. Choosing flying hospitals in the fight against the COVID-19 pandemic: structuring and modeling a complex problem using the VFT and ELECTRE-MOr methods. IEEE Latin America Transactions 2021;19:1099-106. https://doi.org/10.1109/TLA.2021.9451257.

[4] Costa IPA, Maêda SMDN, Teixeira LFHSB, Gomes CFS, Santos MD. Choosing a hospital assistance ship to fight the covid-19 pandemic. Revista de Saude Publica 2020;54:79. https://doi.org/10.11606/s1518-8787.2020054002792.

[5] Rodrigues MVG, Duarte TE, dos Santos M, Gomes CFS. Prospective scenarios analysis: Impact on demand for oil and its derivatives after the COVID-19 pandemic. Brazilian Journal of Operations \& Production Management 2021;18:e20211215. https://doi.org/10.14488/BJOPM.2021.039.

[6] Moreira MÂL, Gomes CFS, dos Santos M, do Carmo Silva M, Araujo JVGA. PROMETHEESAPEVO-M1 a Hybrid Modeling Proposal: Multicriteria Evaluation of Drones for Use in Naval Warfare. Springer Proceedings in Mathematics \& Statistics. 1st ed., Cham: Springer; 2020, p. 38193. https://doi.org/10.1007/978-3-030-56920-4_31.

[7] Song Z, Yan T, Ge Y. Spatial equilibrium allocation of urban large public general hospitals based on the welfare maximization principle: A case study of Nanjing, China. Sustainability (Switzerland) 2018;10. https://doi.org/10.3390/su10093024.

[8] R Core Team. R: A Language and Environment for Statistical Computing 2021.

[9] Moreira MÂ, de Araújo Costa IP, Pereira MT, dos Santos M, Gomes CF, Muradas FM. PROMETHEE-SAPEVO-M1 a Hybrid Approach Based on Ordinal and Cardinal Inputs: MultiCriteria Evaluation of Helicopters to Support Brazilian Navy Operations. Algorithms 2021;14. https://doi.org/10.3390/a14050140.

[10] Ramachandran KM, Tsokos CP. Mathematical statistics with applications in R. Academic Press; 2020 .

[11] Zanjirani Farahani R, Hekmatfar M, editors. Facility Location. Heidelberg: Physica-Verlag HD; 2009. https://doi.org/10.1007/978-3-7908-2151-2.

[12] Arrumdany AC, Sari PP, Rahmadani P, Lubis AI. Web-Based Geographic Information System (GIS) in Determining Shortest Path of MSME Medan City Using Bellman-Ford Algorithm. Journal of Physics: Conference Series, vol. 1255, IOP Publishing; 2019, p. 12075.

[13] Karive O, Hakimi S. The p-median problems. In: An algorithmic approach to network location problems. SIAM Journal on Applied Mathematics 1979;37:539-60.

[14] Li Q, Zeng B, Savachkin A. Reliable facility location design under disruptions. Computers and Operations Research 2013;40:901-9. https://doi.org/10.1016/j.cor.2012.11.012.

[15] Frederick Hillier, Lieberman G. Introduction to Operations Research. 11th ed. New York: McGrawHill Education; 2020.

[16] Berkelaar M. Lpsolve 2020.

[17] Cheng J, Karambelkar B, Xie Y, Wickham H. Leaflet 2021. 